\title{
ESTUDO DE MATÉRIAS-PRIMAS UTILIZADAS EM CERÂMICA VERMELHA DO MUNICÍPIO DE CRATO - CE*
}

Amélia de Santana Cartaxo ${ }^{1}$ Antônio Demouthie de Sales Rolim Esmeraldo²

\section{Resumo}

Este trabalho tem como objetivo o estudo de matérias-primas utilizadas nas indústrias de cerâmica vermelha da Região do Cariri-Ce, de forma a adequar o processamento às especificações de composição, umidade e temperatura para obtenção de componentes com qualidade e características satisfatórias. Para tal, foi realizada a análise de composição através de $\mathrm{FRX}$, limites de Atterberg, retração linear, absorção de água e tensão de ruptura à flexão, pós queima, em três temperaturas distintas. Pode-se observar que algumas argilas apresentaram composições típicas das utilizadas para a fabricação de produtos da cerâmica vermelha, aumento de tensão de ruptura e retração com a temperatura de queima, redução da absorção com o aumento da temperatura de queima e a penas as amostras AM e BP encontraram-se no intervalo de extrusão satisfatória.

Palavras-chave: Estudo; Matérias-Primas; Cerâmica Vermelha, Crato.

\section{STUDY OF RAW MATERIALS USED IN INDUSTRY OF RED CERAMIC FROM CRATO - CE}

\section{Abstract}

This work aims to study the raw materials used in red ceramic industries in the Cariri -Ce Region, in order to adjust the processing compositional specifications, humidity and temperature to obtain components with quality and satisfactory characteristics. To this end, the composition was performed by XRF analysis, Atterberg limits, linear shrinkage, water absorption and flexural stress at rupture, after burning at three different temperatures. It can be seen that some clays showed typical compositions from those used for manufacturing of the red ceramic products the increase of rupture stress and decrease in the firing temperature, reduction of absorption with increasing firing temperature and feathers AM samples and BP met in satisfactory extrusion range.

Keywords: Study; Raw materials; Red Ceramic; Crato.

1 Engenharia de materiais, graduação, estudante, engenharia, UFCA, Juazeiro do Norte, Ceará, Brasil.

2 Engenharia de materiais, graduação, estudante, engenharia, UFCA, Juazeiro do Norte, Ceará, Brasil. 


\section{INTRODUÇÃO}

As argilas são materiais heterogêneos, com variedades de características de região para região. Assim, faz- se necessário à sua identificação, através de composição e propriedades, de forma a melhorar o processamento e o desempenho das peças obtidas.

O município de Crato possui uma grande quantidade de indústrias de cerâmica vermelha com uma grande produção de blocos e telhas, entretanto, as jazidas das quais são extraídas as matérias-primas não são e devidamente estudadas.

O processamento ocorre de forma empírica com a mistura de matérias-primas $A$ composição da mistura utilizada é bastante variável e depende do tipo de argila, do tipo de produto que se deseja fabricar e das próprias características das argilas na frente de lavra. Entretanto, não existe na literatura técnica-científica informações a respeito da otimização desta mistura.

Portanto, esse trabalho tem como objetivo o estudo de matérias-primas representativas utilizadas no município de Crato, através da composição química, propriedades de plasticidade e comportamento sob processo de extrusão, além de propriedades físico-mecânicas de forma a desenvolver produtos com características satisfatórias desde a preparação dos insumos ao processamento propriamente dito de acordo com a normas técnicas

\section{MATERIAIS E MÉTODOS}

O material coletado de duas indústrias na Região do Cariri-Ce, foi acondicionado em sacos e nomeado de acordo com as especificações das indústrias: Argila do meio (AM), Barro Branco (BB) e Barro Preto (BP). O mesmo foi submetido ao beneficiamento: secagem em estufa, moagem em moinho de martelos, peneiramento, armazenamento e identificação.

A quantidade obtida em peneiramento (ABNT n200), foi enviada para o Departamento de Engenharia de Materiais da Universidade Federal de Campina Grande - Paraíba, para a realização de análise química a qual consistiu em análise semi-quantitativa através da fluorescência de raios-X por energia dispersiva pelo equipamento da marca SHIMADZU, modelo EDX-720.

Os limites de Limite de Liquidez, Limite de Plasticidade e Índice de Plasticidade, segundo (1) e (2) respectivamente. O limite de liquidez foi calculado pela equação (1), da American Society for Testing Materials, para o caso de não se obter a condição estipulada pelo método para as 25 pancadas, em que WN é percentagem de umidade correspondente a $\mathrm{N}$ pancadas.

$$
L L=W N *\left(\frac{N}{25}\right)^{0,12}
$$

Corpos-de-prova em formato retangular foram confeccionados em número de 15 com apenas três das amostras, divididos em grupos de 5 e, posteriormente queimados nas temperaturas $700^{\circ} \mathrm{C}, 850^{\circ} \mathrm{C}$ e $1150^{\circ} \mathrm{C}$. Após a queima foram realizados os testes de flexão de três pontos, retração linear e absorção de água.

$O$ ensaio de flexão, foi realizado pela máquina do modelo Flexi 1000 LX-650 da marca Gabbrielli, satisfazendo as condições de exigência. A distância dos apoios foi determinada em 59,3 $\mathrm{mm}$.

$$
T R=\frac{3 Q d}{2 * L * E^{2}}
$$


Após o ensaio de flexão, uma das partes que se rompeu foi colocada em água, permanecendo por $24 \mathrm{~h}$, obtendo-se o peso úmido. A absorção de água (AA) foi encontrada pela equação 6 , em que $\mathrm{Mu}$ é a massa do pedaço da amostra permaneceu dentro da água por $24 \mathrm{~h}$ e Ms é o valor da massa do pedaço da amostra quebrada após o ensaio de flexão.

$$
A A=\frac{M_{u}-M_{S}}{M_{S}} * 100 \%
$$

\section{RESULTADOS E DISCUSSÃO}

As composições químicas e a relação entre óxidos das amostras estudadas estão descritas na tabela1. Pode-se observar que as argilas nomeadas como AM, BP apresentaram composições típicas das utilizadas para a fabricação de produtos da cerâmica vermelha (Souza Santos).

A cor dos produtos estruturais varia principalmente com o estado de oxidação do ferro. A cor dos produtos estruturais varia principalmente com o seu estado de oxidação. Quando se encontra na forma trivalente $\left(\mathrm{Fe}_{2} \mathrm{O}_{3}\right.$, Hematita), com $5 \%$ ou mais os produtos resultantes terão cor vermelha, as argilas de queima branca apresentam menos de 1\%. Assim as quantidades encontradas nas amostras AM e BP são suficientes para resultar em cor vermelha, para amostra BB provavelmente não resultará em cor vermelha (4).

Os óxidos alcalinos $\left(\mathrm{K}_{2} \mathrm{O}+\mathrm{Na}_{2} \mathrm{O}\right)$ agem como fundentes quando presentes em determinada composição, reduzem a temperatura de queima e a porosidade do produto. Estas duas condições são importantes para produtos como os de cerâmica vermelha, pois além de baixar o custo, reduzem a absorção de água e aumentam a resistência mecânica (5). Não se observou nenhuma quantidade de $\mathrm{Na}_{2} \mathrm{O}$.

Os óxidos alcalinos terrosos $\mathrm{MgO}$ e $\mathrm{CaO}$ também podem ter a função de fundentes durante a queima. Eles reagem com fases amorfas e formam fases cristalinas que são mais estáveis frente a umidade (6).

A relação de $\mathrm{SiO}_{2} / \mathrm{Al}_{2} \mathrm{O}_{3}$ variou entre $2,44 \%$ a 2,67\%, dessa forma, as amostras estudadas não estão próximas à caulinita teórica, pois quanto mais próxima esta relação estiver de 1,18 , maior será a quantidade de caulinita presente na argila (7).

Tabela 1. Composição química das amostras (FRX).

\begin{tabular}{|c|c|c|c|c|c|c|c|c|c|}
\hline \multirow{2}{*}{ AMOSTRA } & \multicolumn{7}{|c|}{ CONSTITUINTE (\% em peso) } & \multirow{2}{*}{$\mathrm{SiO}_{2} / \mathrm{Al}_{2} \mathrm{O}_{3}(\%)$} \\
\cline { 2 - 10 } & $\mathrm{SiO}_{2}$ & $\mathrm{Al}_{2} \mathrm{O}_{3}$ & $\mathrm{Fe}_{2} \mathrm{O}_{3}$ & $\mathrm{~K}_{2} \mathrm{O}$ & $\mathrm{MgO}$ & $\mathrm{CaO}$ & $\mathrm{TiO}_{2}$ & Outros & \\
\hline AM & 60,14 & 23,17 & 7,4 & 3,77 & 3,16 & 0,61 & 1,04 & 0,71 & $2,60 \%$ \\
\hline BB & 67,44 & 25,44 & 2,81 & 1,78 & 0,97 & 0,38 & 0,86 & 0,31 & $2,65 \%$ \\
\hline BP & 62,46 & 23,37 & 5,98 & 2,78 & 2,44 & 0,98 & 0,97 & 1,01 & $2,67 \%$ \\
\hline
\end{tabular}

Os limites de Atterberg estão listados na tabela 2. O método de Atterberg determina o intervalo em que a argila pode ser moldada, de acordo com o índice de plasticidade que é a diferença entre o limite de liquidez, ou umidade acima da qual uma massa não é moldável, e o limite de plasticidade, ou umidade mínima para uma massa ser moldável. $O$ índice de plasticidade IP define-se como a diferença entre o LL e LP (8). 
Observou-se que o limite de liquidez das amostras variou entre 27,3 e $46,1 \%$ e o Limite de plasticidade varioe de 7,8 a $23,1 \%$. Com base na literatura verifica-se que dos valores obtidos (9) apenas a amostra BB não está dentro do intervalo para cerâmica vermelha.

Após análise do conjunto de dados obtidos pelo aparelho de Casagrande, segundo critérios utilizados em mecânica dos solos (10), massas com presença de argila podem ser classificadas como se segue: fracamente plástica $(1 \%<\mathrm{IP}<7 \%)$, mediamente plásticas $(7 \%<\mathrm{IP}<15 \%)$,altamente plásticas, $(\mathrm{IP}>15 \%)$. De acordo com esse critério todas as amostras estudadas são altamente plásticas.

Considera-se na literatura que as argilas ou massas argilosas devem apresentar um IP mínimo de $10 \%$, valores menores podem ser problemáticos para 0 processamento cerâmico, pois se encontram dentro dos limites de tolerância utilizados pelas indústrias, podendo resultar em mudança na consistência da argila ou massa a argilosa (7), assim, as amostras estudadas obedeceram a esse critério.

Tabela 2. Resultado do limite de Atterberg.

\begin{tabular}{|c|c|c|c|}
\hline Amostra & Limite de Liquidez (LL) & Limite de Plasticidade (LP) & Índice de Plasticidade (IP) \\
\hline AM & $46,1 \%$ & $23,1 \%$ & $23,0 \%$ \\
\hline BB & $27,3 \%$ & $7,8 \%$ & $19,5 \%$ \\
\hline BP & $35,7 \%$ & $18,2 \%$ & $17,5 \%$ \\
\hline
\end{tabular}

De acordo com os valores de índice de plasticidade e limite de plasticidade pode classificar as argilas com base no seu comportamento mediante extrusão. Na extrusão aceitável a argila ou massa argilosa deve apresentar IP entre 10 e $34 \%$ e o LL 18 entre e 31\%. No entanto, para uma extrusão ótima, o IP deve estar entre 15 e $25 \%$ e o LP entre 18 e $25 \%$ (11).

A figura 1 apresenta adequabilidade à extrusão na qual, observa-se que a amostra BP encontra- se na extrusão ótima já a argila AM localiza-se dentro da extrusão aceitável (mas relativamente perto da zona ótima. Por outro lado, a amostra BB encontra-se fora das zonas de extrusão, com baixo índice de plasticidade provavelmente devido ao seu maior conteúdo de $\mathrm{SiO}_{2}$ podendo representar uma maior fração areia.

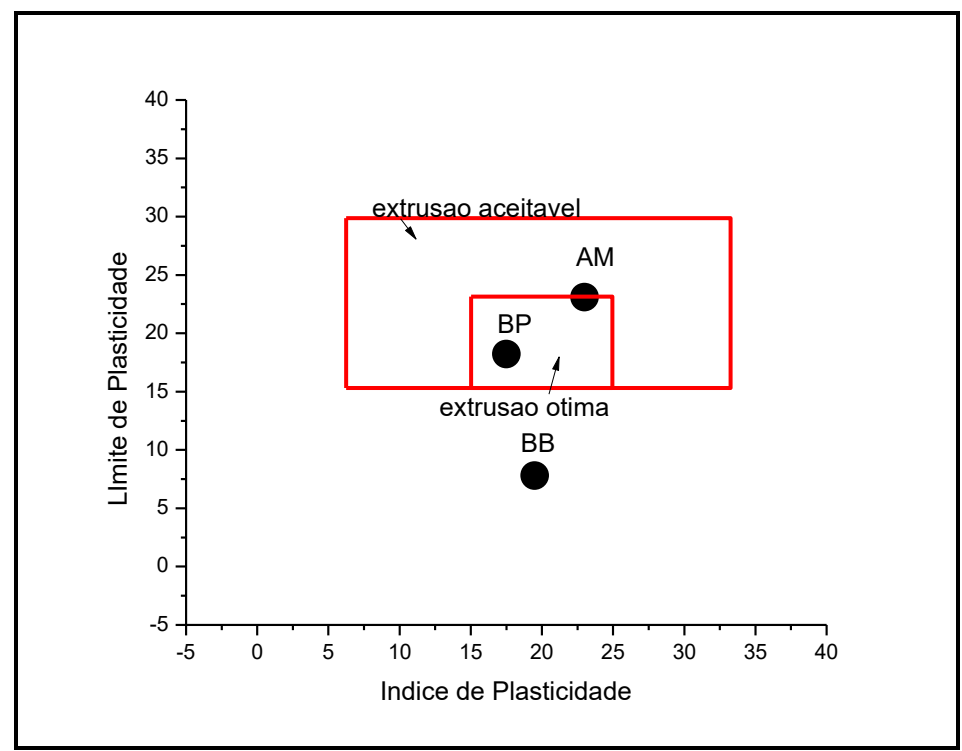

Figura 1: Prognóstico de extrusão. 
A figura 2 apresenta a tensão de ruptura dos corpos de prova confeccionados com as argilas. De acordo com a figura 2, houve um aumento da tensão de ruptura à flexão para as amostras BB e BP. Apenas as amostras AM queimada a $850^{\circ} \mathrm{C}$ e $700^{\circ} \mathrm{C}$ e BP queimada a $1050^{\circ} \mathrm{C}$ atingiram o valor esperado para resistência de blocos (acima de 5,5Mpa).

A amostra AM também apresentou aumento de tensão até a queima em $850^{\circ} \mathrm{C}$, em $1050^{\circ} \mathrm{C}$ ocorreu um decréscimo da tensão, devido à expansão dimensional a qual se deve, provavelmente, a presença de "coração negro". Segundo a literatuta (12), este defeito é causado pela presença de matéria orgânica que geralmente acompanha as argilas, e que durante a queima não sofre combustão completa e deixa no interior do corpo queimado, uma fuligem escura característica. Desta maneira, os resultados das propriedades físico-mecânicas obtidas nos corpos de prova queimados à temperatura de $1050^{\circ} \mathrm{C}$, para esta amostra, não são confiáveis.

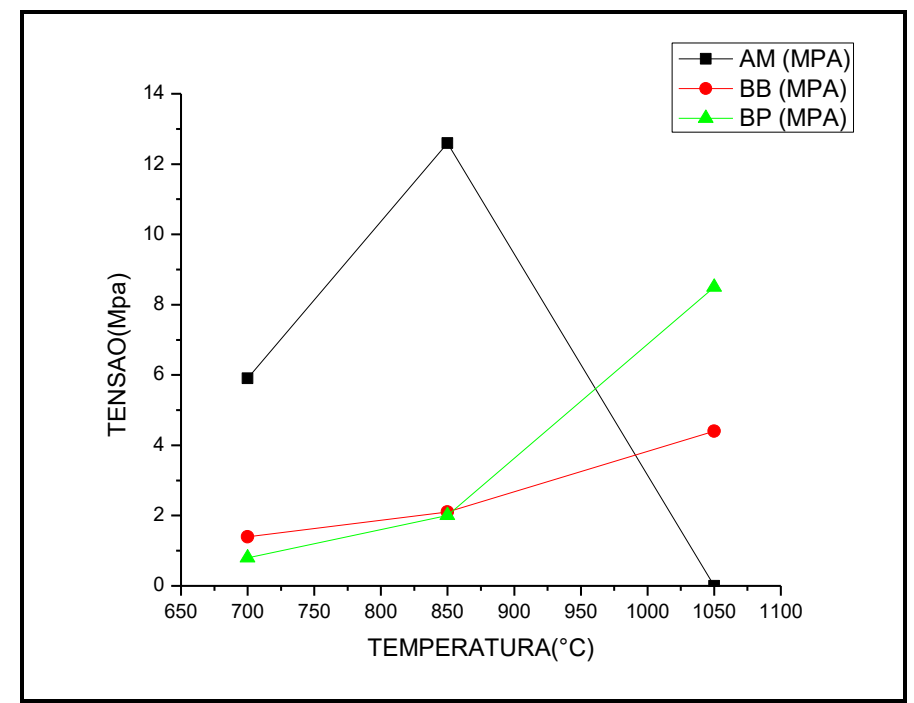

Figura 2. Tensão de ruptura por flexão após queima.

A figura 3 apresenta os resultados para a absorção de água. De acordo com o gráfico, houve uma redução da absorção de água com o aumento da temperatura de queima. A absorção de água deve ser inferior a $25 \%$ para os corpos de prova de argilas para produção de blocos cerâmicos, verifica-se que todas as amostras apresentaram valores de acordo com o indicado 


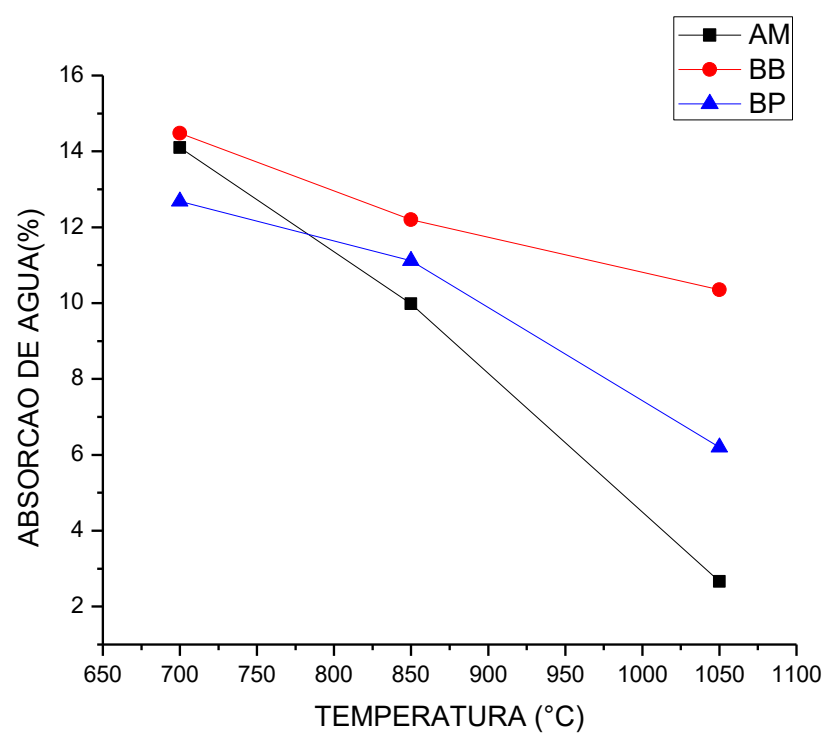

Figura 3: Absorção de Água

A figura 4 mostra a retração linear de queima. É possível notar que houve um aumento da retração com o aumento da temperatura de queima, mostrando que a sinterização está progredindo, e que também foi obtido por (13) e (14). A amostra $\mathrm{AM}$ apresentou maior porcentagem de retração entre o intervalo de $700^{\circ} \mathrm{C}$ a $850^{\circ} \mathrm{C}$.

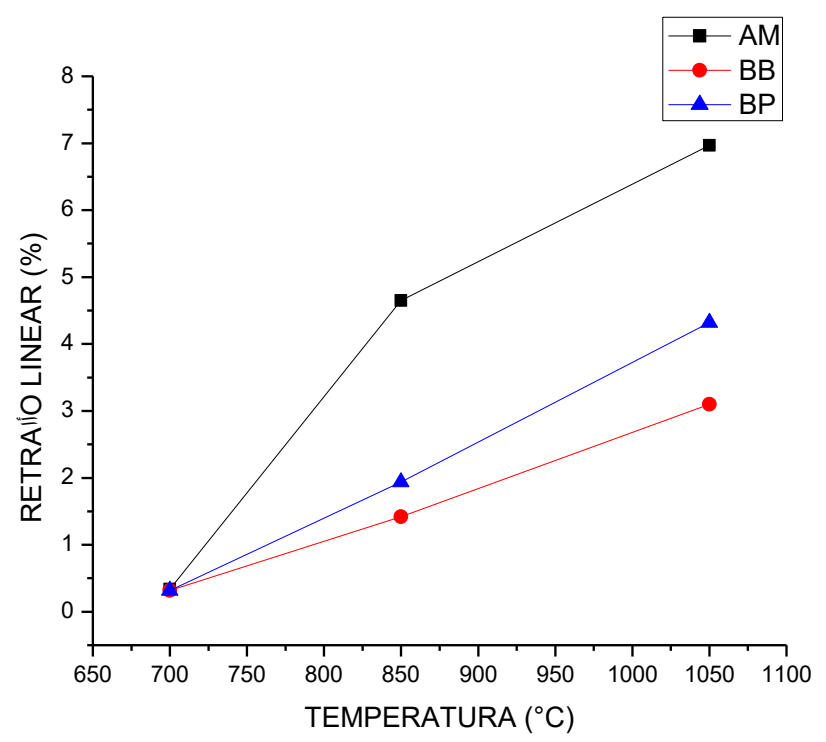

Figura 4: Retração Linear após queima.

\section{CONCLUSÃO}

Foram estudadas quatro de tipos de matérias-primas utilizadas em cerâmica vermelha as quais de todas as amostras apenas AM e BP possuem composições típicas para cerâmica vermelha, com altas porcentagens de sílica, alumina e óxido de ferro.

De acordo com o prognóstico de extrusão apenas as amostras AM e BP encontramse em intervalos para execução de extrusão sem aditivos. 
Para o ensaio de ruptura à flexão apenas as amostras BB e BP, o valor da tensão cresceu com o aumento da temperatura de queima. A amostra AM apresentou "coração negro" após a queima em $1050^{\circ} \mathrm{C}$ que fragilizou o corpo de prova, consequentemente reduziu a tensão de ruptura.

Para todas as amostras a absorção de água reduziu e a retração linear aumentou com o aumento da temperatura de queima.

\section{REFERÊNCIAS}

1 ABNT (Associação Brasileira de Normas Técnicas). NBR 6459: Determinação do limite de liquidez-método de ensaio. Rio de Janeiro: ABNT, 1984.

2 ABNT (Associação Brasileira de Normas Técnicas). NBR 7180: Determinação do limite de plasticidade- método de ensaio. Rio de Janeiro: ABNT, 1984.

3 Santos P S. Ciência e Tecnologia de Argilas v. I, São Paulo: Edgard Blücher.1992.

4 Murray H H. Applied Clay Mineralogy: Occurrences, Processing and Application of Kaolins, Bentonites, Palygorskite-sepiolite, and Common Clays. Oxford: Elsevier; 2007.

5 Associação Brasileira de Cerâmica [acesso em 14 de abril de 2016]. Disponível em: http://www.abceram.org.br/site/?area=4\&submenu=47.

6 E, Facincani. Tecnologia Cerâmica: los Ladrillos, Faenza Ed. Iberica S. L. Editora, Barcelona, Espanha, 1993 p. 263.

7 Vieira C M F, Pinheiro R M. Avaliação de argilas cauliníticas de Campos dos Goytacazes utilizadas para fabricação de cerâmica vermelha. Cerâmica. 2011; (57) 319323.

8 Barba A, Beltràn V, Feliu C, García J, Ginés F, Sànchez E, Sanz V. Materias primas para la fabricación de soportes de baldosas Cerámicas. ITC, 1를. Castellón, Espaňa. 1977.

9 Macedo R S, Menezes RR, G. A. Neves, H. C. Ferreira. Estudo de argilas usadas em cerâmica vermelha. Cerâmica.2008; (54) 411-417.

10 Caputo H P. Mecânica dos solos e suas aplicações fundamentais. Livros Técnicos,vol.1. Rio de Janeiro,1994.

11 Santos C V P, Silva A R, Filho M A S G, Neto E F, Rabelo A A. Índice de Plasticidade e Análise Racional de Argilas de Marabá (PA) para Avaliação das Zonas de Extrusão. Cerâmica Industrial. 2012; 25-17.

12 Macedo, R. S, Santana, L. N. L., Silva, T. D. A., Vasconcelos, S. M. C., Carreiro, M. E. A., Neto, J. L. B., Leite, M. D. R. Caracterização Preliminar de argila vermelhas do Estado da Paraíba ,visando a produção de telhas e blocos cerâmicos. In: Anais do 55은 Congresso Brasileiro de Cerâmica, Porto de Galinhas, PE: 2011

13 Cartaxo A S, Esmeraldo, A D S R, Suassuna P M, Prado A C A, Neiva L S, Brasileiro M I. Estudo e avaliação das propriedades de argilas Utilizadas na indústria de cerâmica vermelha da Região do Cariri- Ceará, In: CONGRESSO ANUAL DA ABM -

INTERNACIONAL, 70., 2015, Rio de Janeiro. Anais eletrônicos. São Paulo: Associação Brasileira de Metalurgia, Materiais e Mineração, 2015. Disponível em: http://www.abmbrasil.com.br/anais. Acesso em: 14 de abril de 2016.

14 Maestrelli S C, Roveri C D, Nunes A G P, Faustino L M, Aielo G F, Pinto L P A, Manochio C, Cal T M L, Ribeiro F F, Mariano N A. Estudo de caracterização de argilas não plásticas da região de Poços de Caldas, MG.Cerâmica.2013; (59) 243-248. 